\title{
Multidimensional Interpretation of Speech Indirectness
}

\author{
Maoxiang $\mathrm{Ma}^{1} \&$ Yingxia $\mathrm{Li}^{2}$ \\ ${ }^{1}$ School of Foreign Languages, Shandong Normal University, Jinan, China \\ ${ }^{2}$ Laiwu Fengcheng High School, Laiwu, China \\ Correspondence: Maoxiang Ma, Confucius Institute at Central Connecticut State University, Diloreto Hall Room \\ 015, 1615 Stanley Street, New Britain, CT 06050, USA. E-mail: mamaoxiang@126.com
}

Received: January 22, 2016 Accepted: February 13, 2016 Online Published: February 26, 2016

doi:10.5539/ells.v6n1p133 URL: http://dx.doi.org/10.5539/ells.v6n1p133

\begin{abstract}
Indirect speech is a universal phenomenon of human language communication. Out of courtesy or some other social conventions, people convey their intentions not always in a straightforward manner, but often by implication, expressing subtle implication politely. Thus, the proper interpretation and understanding of indirect speech is vital to our success of communication. This paper reviews the predecessors' interpretation from the Anglo-American tradition of pragmatic theory, relevance theory, adaptation theory to other linguistic perspectives with a brief comment on those dimensions, and at the same time, it tries to explore a comprehensive interpretation approach to indirect speech, in order to enrich the mechanism of indirect speech interpretation, and help with successful communication.
\end{abstract}

Keywords: indirectness; indirect speech act; interpretation

\section{Introduction}

Indirect use of language is a universal phenomenon in human communication. In real language practice, in most cases, people do not transmit their intentions directly to the hearer, but take a more indirect way. This is sometimes due to certain restrictions of social conventions, sometimes for specific communicative effect, with a deliberate omission of some words to hide the information between the lines, thus making an information gap. The example "Do you want more tea?" is in the form of a question asking whether the hearer would like to have some more tea, but in a specific context, like a visitor has stayed long enough and the host has something at hand to handle and he might hint or remind the visitor: "It's time for you to leave". Such function is not inherent in the meaning of a sentence, but involves an interpretation of the contextualized speech, shared background knowledge and reasoning ability of the hearer (Fang \& Li, 1996).

There are two different situations in people's use of indirect speech (He, 2000, p. 19). One is the speaker, for some reason, chooses to take an indirect way of speaking when he can use direct speech, which may bear a particular implication, or implicature, as Grice called it. Another case is the speaker can not express themselves directly, so he has to rely on the use of indirect means of expression. Indirect speech can also be interpreted in the light of the relation between the literal meaning and utterance meaning of the speech. When these two meanings are inconsistent, the use of the speech is indirect (He, 2000).

The Indirectness of speech is closely related to the implied pragmatic information in the given context. There is a multifold of manifestations of this indirectness, the most prominent of which is to convey indirect meaning through indirect speech acts. Indirect speech act implies the speaker's real communicative intention, which is a deviation from the literal meaning of the speech, and the gap the communicative intention and the literal meaning gives rise to indirectness of speech. Therefore, the author of the article will ignore the difference between indirectness of speech and indirect speech acts. Meanwhile, in the multidimensional interpretation of indirect speech later on in the study, different scholars have used expressions with little difference, such as: conversational meaning, illocutionary meaning, hidden meaning, and so on, and the essence of those different expressions share the same property, so the difference between them is ignored.

\section{Major Interpretation Dimensions}

The phenomenon of indirect use of speech has aroused the interest and the attention of some linguists, who have probed this phenomenon from different perspectives. To date, scholars have studied the phenomenon and tried to 
interpret indirect speech from cognitive perspective, adaptation perspective as well as the traditional Anglo-American pragmatic perspective including indirect speech act theory, the principle of conversational cooperation and implication, politeness principle etc.

\subsection{Traditional Anglo-American Pragmatic Perspective}

Indirect use of English language is very common and frequent, and many Anglo-American linguists have done much research in this area. The earliest interpretation along this line is by British philosopher Austin, who proposed the famous trichotomy of every indirect speech act, the core of his speech act theory: locutionary act, illocutionary act and perlocutionary act. By performing a speech act, the speaker can produce a special intended communicative effect in the hearer, which is the implication of such indirect speech act.

Later, the famous American philosopher Searle combined the speech act theory and Grice's conversational implicature theory to establish the necessity of inference and reasoning and proposed the indirect speech act theory. The advantages of the inference theory is that it distinguishes between two levels of meaning---literal meaning and conversational meaning and presumed a series of step-by-step inference steps. Depending on a number of contextual factors, pragmatic knowledge and inference ability, the hearer can reasonably deduce the implication that a speech act has in a particular context (Fang \& Jiang, 2002). But this theory is not fully explanatory, because whether both the speaker and the hearer can share background knowledge and contextual information is yet questionable. Different people have different means of cognition, so absolute sharing of knowledge is impossible, it's just an idealized state. Meanwhile, in the real language communication, people do not really follow these steps to infer the speaker's communicative intention; it's rather a subconscious act to approach, to a maximum extent, the communicative intention on the basis of negotiation. Xiong (2007) also noted that the implementation and interpretation of speech acts should be based on the specific cultural constraints and discourse turns as the conversation goes on. According to the definition of indirect speech acts, the literal meaning is not inappropriate in a particular context, but just not sufficient. Therefore, it is entirely possible for the hearer to understand the seemingly correct literal meaning and just stop at that. In this case, how does the speaker convey yet another level of indirect conversational intention? Searle's theory seems weak to explain this.

Grice proposed the concept of implicature in 1967, presenting insightful ideas, from the perspective of the cooperative principles to be followed in conversation, on understanding the speaker's intention and the felicity conditions and the process of inference as well as the nature of the information conveyed. Grice's theory of implicature comprehensively reveals the non-verbal communication conventions that are closely related with verbal communication. And it enables a study of the real communicative meaning of an utterance according to the context rather than a study of the language itself from within language system, thus providing a functional explanation for the language facts, where some other language theories falter (Fang \& Jiang, 2002). Those principles must be followed in principle, but in language practice, it is also common for the principles to be deliberately violated. The Emphasis of Grice's theory is not placed on the following of the principles, but more on the different cases in which the maxims of the principles are violated to produce implicature. If the speaker violates a maxim on the basis of following principle of communicative cooperation, and believes the hearer has the ability to detect and understand, then the purpose of this ostentatious violation of a certain maxim is to express certain illocution. If people violate the principles in order to generate conversational implicature, are these principles essential to exist? According to the principles of cooperation, the most direct expression of speech is most effective for communicative purposes. Why do the cooperation-based communicators deliberately violate the maxims of cooperative principles $(\mathrm{CP})$ ? In this sense, this theory does not explain why people would use indirect speech fundamentally. Grice's (1975) system of maxims is intended to reflect the general laws of human language communication. In addition, his proposed theory does not specify what specific criteria apply, such as the quantity in the maxim of quantity, how much is the right quantity of information and how much is the overloaded information? And there are repetition in content and haziness in operation, which also foreshadows the subsequent birth of the relevance theory. If the $\mathrm{CP}$ well explains implicature of indirect speech acts, then such pragmatic meanings as social, emotional etc. are not fully explained. Finally, in a different cultural background, the applicability of the CP and its maxims is different. Keenan (1975) has pointed out that the CP is not universal criterion governing and restricting people's conversations (He \& Ran, 2009). Therefore, CP ignores the important cultural factor that affects communication, and interpretation of indirect speech acts is in some cases biased.

For the above theoretical limitations, some people henceforth resorted to politeness to explain this problem. Brown and Levinson (1987) believe that in the communication process, cooperation is not necessarily the first consideration, but appropriateness, namely politeness, is. Just out of consideration of politeness, people will take 
different pragmatic strategies in performing different speech acts, and sometimes have to take an indirect way to express their true intentions in order to maintain faces of both sides. The major consideration of performing a speech act in an indirect way for purpose of politeness is mainly on account of bilateral relations and social status differences. So in this sense, what Brown and Levinson proposed the notion of face helps reveal the social significance of discourse, but not all can be explained by the social significance. There is more complex social implication when one is "playing it safe for self-preservation" for some other considerations (Chen, 1999). Later Leech (1983) put forward the principles of politeness (PP) to explain why people choose to use indirect speeches, but fail to explain how people infer the conveyed implication by an indirect speech act. On the other hand, not all indirectness is out of politeness, some other factors also govern and restrict people's choice of language. Liu Guohui (2001) noted that in addition to PP, other factors like economy principle, aesthetic appeal, human relationship, white lies, limitlessness of dynamic communicative presupposition and unspecified situation etc. all act as hidden motives to the usage of indirect speech act.

\subsection{Cognitive Perspective}

Since the 1980s, the cognitive study of language function is becoming an increasingly important part of cognitive linguistics. The most influential is the relevance theory in Sperber and Wilson's monograph "Relevance: Communication and Cognition" (1986a, 1995). The authors argue that the goal of human cognition is to arouse people's attention to relevant information. To communicate is to imply that the information communicated is relevant. So relevance is regarded as the key to human communication and cognition. What is the most fundamental for human communication is relevance. The speaker and hearer seek maximum relevance and optimal relevance in constant negotiation. Communication is also considered a cognitive process, the success of which depends much on the shared cognitive contextual environment and recognition of this, and as the communication goes on the cognitive contextual environment is also undergoing constant dynamic changes. While understanding the context of the discourse acts as a constraint, it provides only objective external condition. The dynamic factor that really works is the ability of human perception of the world, and the objective factors need the cognitive processing of discourse in order to produce meaning. And the success of understanding the meaning involves also pragmatic reasoning or inference based on the background or contextual information. Pragmatic reasoning should not only focus on the hidden meaning of communication, but also be concerned about the apparent meaning of communication. The hidden meaning here is different from Grice's implicature in that it includes not only the implicit premise, but the implicit conclusion, while the Grice's implicature theory can only deduce premise.

Obviously, relevance theory explained more fully than the previous theories the understanding of the indirect speech, but there are also problems with the theory. Firstly, according to theory, relevant information in verbal communication is derived from an inference of the discourse combined with cognitive contextual assumptions. However, how does the listener expand the schema of assumptions, starting from the initial assumptions and making the appropriate choice from a range of cognitive context? How are these series of reasoning assumptions produced, and what's the order of production? How can the hearer select the most relevant information, among a large quantity? Secondly, the relevance is a relative concept, depending on the cognitive effort the hearer makes and possible contextual effect obtained by inference, as the optimal relevance is that derived by minimal cognitive effort to maximize contextual effect of cognitive inference. So how does the listener negotiate in between? How to determine the minimal cognitive effort and the maximal contextual effect? These are not clearly defined here.

\subsection{Adaptation Perspective}

Another new pragmatic theory-Adaptation Theory was put forth by Verschueren in 1990s. According to Verschueren, the use of language is a choice of the language, but people's choice of language is not arbitrary, but based on some pragmatic principle - context. Due to the variability, negotiability and adaptability of language, adaptation to contextual factors can happen at all levels of language, as the language user adapts to the objective world, the social world and mental world. Language adaptability involves four aspects: contextual adaptability, structural adaptability, dynamic adaptability and salience of adaptability. Language adaptability allows language users to make a flexible choice from different language options, thus maximizing the needs of communication.

Chen Xinren (2001) considered the language adaptation theory is based on a conversational implicature, politeness principle, face theory, the theory of speech act. On the one hand, it explores the adaptation features that occur at all levels of the discourse, revealing the mechanism for the linguistic adaptation to occur; on the other hand, it provides a theoretical framework to investigate any pragmatic phenomenon. Yu Guodong (2004) made an interpretation of indirect speech acts on the basis of language adaptability: Choice of indirect speech act 
is an adaptation to the language reality; Choice of indirect speech act is an adaptation to social conventions; Choice of indirect speech act is an adaptation to psychological motivation. Indeed, the language adaptation theory gives a comprehensive interpretation of indirect speech from all levels of language, which makes it very operational. However, there are some limitations with the theory: Adaptation theory has a strong generality, however, in fact, due to the complexity of human society, language users are not all the time making language choices in accordance with the adaptation theory. Zero adaptation phenomena also exist, as when the speaker persists in his own way, without considering the social and cognitive constraints. So adaptation is a relative concept; for different language users, on different occasions, the degree of linguistic adaptation is different. In addition, different cultures require different language adaptation, and there is a lack of sufficient evidence to support any discussion in this regard.

\subsection{Other Perspectives}

Besides the above pragmatic perspectives, there are also some linguistic researchers trying to put forward their own unique insights into the expression of certain function in another form in communication from their own perspective with the help of discourse linguistics. Fang Liqing and Jiang Weiqing (2002) illustrated the illocutionary function relationship between sentences from the perspective of discourse analysis of coherence, holding that links between sentences on propositional content lie in relations of illocutionary act between sentences. Coherence is the connection between sentences in illocutionary function. Successful communication depends on the participants' understanding and inference of propositional illocutionary function on the basis of context, keeping discourse coherence, and quick repair when coherence is interrupted. In common with pragmatic study, discourse analysis manages to explain the coherence of the natural language, however, its identification means that the presupposed and implicit information is revealed. Wang Lingling and Gu Ying (2010) interpreted indirect speech act from the perspective of metaphors of mood in systemic functional linguistics, and argued that indirect speech act results from inconsistency between the language form and its function due to inconsistency between tone and function caused by the metaphors of mood. Therefore, the correct understanding of indirect speech act depends on the understanding of tone as well as context. The above two factors make it possible to integrate pragmatics and other linguistic theories, which provides a new perspective for the interpretation of indirect speech.

\section{Integrative Perspective}

In everyday communication, the common phenomenon that one often does not mean what one says brings people to realize that signs and symbols do not necessarily transmit what the signs and symbols mean but by means of these signs and symbols, the communicators convey what they intend to mean, or the communicators' intention. Therefore, to ensure smooth communication, it is extremely necessary to have a clear idea of the production mechanism of indirect speech. Through the above analysis, we found that there are certain degrees of limitations for each of the previous theories. Because the indirectness of indirect speech is a complex contradiction, involving all aspects of the factors, the most reasonable interpretation should be a comprehensive one, integrating each theory's advantages, both from the pragmatic perspective of the language, and from cognitive relevance and language adaptation, rather than absolute interpretation by any single theory, namely, PP and adaptation theory can be used to analyze the indirect speech production mechanism; pragmatic inference, relevance theory, and adaptation theory can be used for interpretation of indirect speech. In the process of verbal communication, these theories affect people in one subconscious way or another. The impact of different cultural backgrounds on speech understanding cannot be overlooked, either. Here the author's perspective can be illustrated by resorting to the most classic example proposed by Searle.

\section{Student X: Let's go to the movies tonight.}

\section{Student Y: I have to study for an exam.}

In light of people's general cognitive experience, to the invitation from student $\mathrm{X}$, there are only two alternative responses for student $\mathrm{Y}$, either accept or refuse the invitation, but it seems that the response of student $\mathrm{Y}$ does not belong to either of the alternatives. However, student Y's response does make sense and his intention is well conveyed by it. How does student $\mathrm{Y}$ achieve this? And how does student $\mathrm{X}$ interpret $\mathrm{Y}$ 's real conveyed intention? Firstly, student $Y$ chooses to respond student $X$ in this indirect speech act because student $Y$ wants to adapt to the dynamic process of his psychological world as well as the hearer's, the speaker does not want to threaten the hearer' $\mathrm{s}$ face by using the polite expression. Such an indirect expression can help maintain face of both sides while conveying the speaker's intention. Student $\mathrm{X}$ will first make cognitive reasoning on student $Y$ 's response, considering that Y's response should possess the maximal relevance to X's invitation though not directly answering X. According to Y's explicit speech "I have to study for an exam". X collects contextual information, 
adjusting his cognition to context to deduce "Y won't go to the movie, because he needs to study for an exam". And hence, communicative intention becomes clear to both sides. Here the interpretation of the indirect speech act by $\mathrm{Y}$ does not involve the influence of culture, and therefore negligible, but in cross-cultural communication, cultural factors can not at all be neglected.

\section{Summary}

Diversity in language expressions makes our life more colorful, but also makes interpersonal communication complicated, and also makes indirect speech a topic of great interest for great many scholars. In addition that the "roundabout" indirect expression is commonplace in communication, so a thorough knowledge of the theoretical basis and production mechanism of speech indirectness is critical for successful communication. The author of this article summed up the interpretations made so far to indirect speech act from the traditional Anglo-American pragmatic perspective, cognitive perspective, adaptation theory and other perspectives, based on which, an integrative perspective was proposed, hoping that it can help interpret the different language phenomenon and different stages of language understanding. The Traditional Anglo-American Pragmatic Perspective is easier to operate and interpret some language facts, but more restricted to limited language phenomena, even it cannot well explain the self-contradictory aspect; The Cognitive Perspective is trying to overcome some of the limitations with the traditional perspective, seeking an inner production and interpretation mechanism of language output, but it involves some cognitive misconceptions that needs clarification; The adaptation one, based on contextual factors, is more flexible and has its generality in use, but it also shows that the interpretation of indirectness is context-specific, so an overall knowledge of different cultures and social rites etc. have to be involved; Similarly, some other perspectives are quite limited to a portion of the language facts; The integrative perspective is proposed for greater flexibility, but this interpretation perspective needs more language facts to verify and further research might lead to a more enlightening insight and better understanding of linguistic reality.

\section{Acknowledgments}

Funded Project: Shandong Provincial Social Science Planning Research Project: "Developing Learners' Pragmatic Competence in Foreign Language Teaching" (11BWZJ04) and Shandong Normal University Teaching Reform Project: "Research on Learners' Self-repair Mechanism in Interactive L2 Classroom" (2012035)

\section{References}

Austin, J. L. (1962/1968). How to Do Things with Words. Cambridge, Mass: Harvard University Press.

Brown, P., \& Levinson, S. (1987). Politeness: Some Universals in Language Usage. Cambridge, Cambridge University Press.

Brown, P., Levinson, S., \& Goody, E. (1978). Universals in Language Usage: Politeness Phenomena.

Chen, X. R. (1999). The Pragmatics of Interactional Overinformativeness. Unpublished Dissertation. Guangzhou University of Foreign Studies.

Chen, X. R. (2001). Towards the Full Interpretation Requirement for Pragmatics Accounts. Modern Foreign Languages, (4), 378-389.

Fang, L. Q., \& Jiang, W. Q. (2002). Interpretation of Indirect Speech Act. Journal of Southwest University for Nationalities, 23(9), 256-257.

Fang, L. Q., \& Li, Q. H. (1996). Identification of Values of Public Relations Language. Jiangxi Social Science, 17(8), 53-55.

Grice, H.P. (1975). Logic and Conversation. In P. Cole \& J. Morgan (Eds.), Syntax and Semantics (Vol. 3). New York: Academic Press.

He, Z. R. (1988). A Survey of Pragmatics. Changsha: Hunan Education Publishing House.

He, Z. R. (2007). New Developments in Pragmatics: Relevance, Adaptation, Memetics. Shanghai: Shanghai Education Press.

He, Z. R., \& Ran, Y. P. (2009). A New Survey of Pragmatics. Beijing: Peking University Press.

He, Z. X. (1984). Language Indirectness of English. Journal of Foreign Languages, 31(3), 9-13.

He, Z. X. (1989). Introduction to Pragmatics. Shanghai: Shanghai Foreign Language Education Press.

He, Z. X. (2000). A New Introduction to Pragmatics. Shanghai: Shanghai Foreign Language Education Press. 
Leech, G. (1983). Principles of Pragmatics. New York: Longman.

Levinson, S. (1983). Pragmatics. Cambridge: Cambridge University Press.

Liu, G. H. (2001). An Exploration of the Implicit Motivation of Indirect Speech Act Preference. Journal of Shandong Foreign Language Teaching, 22(4), 49-51.

Searle, J. (1969). Speech Acts. Cambridge: Cambridge University Press. http://dx.doi.org/10.1017/CBO9781139173438

Searle, J. (1975). Indirect Speech Acts. In P. Cole \& J. Morgan (Eds.), Syntax and Semantics (Vol. 3). New York: Academic Press.

Searle, J. (1979). Expression and Meaning. Cambridge: Cambridge University Press. http://dx.doi.org/10.1017/CBO9780511609213

Searle, J. (2001). Expression and Meaning: Studies in the Theory of Speech Acts. Beijing: Foreign Language Teaching and Research Press.

Sperber, D., \& Deirdre, W. (1986). Relevance Communication and Cognition (1st ed.). Oxford: Blackwell.

Sperber, D., \& Deirdre, W. (1995). Relevance Communication and Cognition (2nd ed.). Oxford: Blackwell.

Verschueren, J. (1999). Understanding Pragmatics. London and New York: Arnold.

Wang, L. L., \& Gu, Y. (2010). Analysis on the Indirect Speech Act from the Metaphors of Mood Perspective. Journal of Southwest University of Science and Technology, 27(4), 67-50.

Xiong, X. L. (2007). Inference in Language Use. Shanghai: Shanghai Foreign Language Education Press.

Yu, G. D. (2004). A Study on Code-switching from an Adaptation Theory. Contemporary Linguistics, 6(1), 77-87.

\section{Copyrights}

Copyright for this article is retained by the author(s), with first publication rights granted to the journal.

This is an open-access article distributed under the terms and conditions of the Creative Commons Attribution license (http://creativecommons.org/licenses/by/3.0/). 